\title{
Sterile neutrino dark matter production
}

\author{
Dmitry Gorbunov ${ }^{1, \star}$ \\ ${ }^{1}$ Institute for Nuclear Research of the Russian Academy of Sciences, \\ 60th October Anniversary prospect 7a, Moscow 117312, Russia
}

\begin{abstract}
Sterile neutrinos provide active neutrinos with masses and mixing, and hence is one of the well-motivated candidate for dark matter. We discuss the sterile neutrino production mechanisms operating in the early Universe and show that additional scalar coupled to sterile neutrino can significantly change the situation, making moderate sterileneutrino mixing and small sterile neutrino masses consistent with current cosmological and astrophysical bounds. Further searches for a narrow line in galactic $X$-rays and even direct searches for $\mathrm{keV}$-scale sterile neutrinos in particle physics experiments can probe the suggested setup.
\end{abstract}

\section{Introduction}

Charged fermion sector of the Standard Model of particle physics (SM) exhibits perfect symmetry between left-handed and right-handed particles. The rest of the fermions, neutrinos, drastically violate this symmetry. All three neutrinos, electron $v_{e}$, muon $v_{\mu}$ and $\tau$-neutrino $v_{\tau}$, are left-handed fermions, while their antiparticles, $\bar{v}_{e}, \bar{v}_{\mu}$ and $\bar{v}_{\tau}$, are right-handed. The absence of right-handed neutrino counterparts is inherent characteristic of the SM, where neutrinos are the only massless fermions. Thus, neutrino sector in the SM is exceptional from the theoretical point of view.

In the SM the neutrino sector is exceptional also from the phenomenological point of view. Indeed, this is the only place where the established theory, SM, fails to explain results of particle physics experiments. The phenomenon is called neutrino oscillations and consists in changing the neutrino type, called flavor, while the particles propagate between the source (neutrino emitter) and detector (neutrino absorber).

One can describe the oscillations assuming that neutrinos are actually massive particles, with masses $m_{i}, i=1,2,3$, and the flavor basis, $\left|v_{\alpha}\right\rangle, \alpha=e, \mu, \tau$, and mass basis, $\left|v_{i}\right\rangle$, are not aligned. The two bases are related through the unitary $3 \times 3$ mixing matrix, dubbed Pontecorvo-Maki-NakagawaSakata (PMNS) matrix $\hat{U}$ as follows,

$$
\left|v_{i}\right\rangle=U_{\alpha i}\left|v_{\alpha}\right\rangle .
$$

Then, in the flavor basis the elements of neutrino mass matrix $\hat{M}$ are defined as

$$
M_{\alpha \beta}=\left\langle v_{\alpha}|\hat{M}| v_{\beta}\right\rangle=\left(\hat{U} \hat{M}^{(m)} U^{\dagger}\right)_{\alpha \beta}, \quad \text { where } \quad M_{i j}^{(m)}=m_{i} \delta_{i j},
$$

that is how the neutrino mass matrix looks in the mass basis: it is diagonal by definition of the basis.

^e-mail: gorby@ms2.inr.ac.ru 
In its rest-frame a neutrino mass eigenstate evolves in time (from zero to $t$ ) as any other mass state does,

$$
\left|v_{j}(t)\right\rangle=\mathrm{e}^{-i m_{j} t}\left|v_{j}(0)\right\rangle
$$

Likewise, in the laboratory frame, neutrino evolves in time and propagates in space (covering the distance $L$ ) according to

$$
\left|v_{j}(t, L)\right\rangle=\mathrm{e}^{-i\left(E_{j} t-p_{j} L\right)}\left|v_{j}(0)\right\rangle,
$$

where $E$ and $p$ are energy and 3-momentum, respectively. Remarkably, in all experiments with neutrinos, they are produced being ultrarelativistic, so one can expand $p=\sqrt{E^{2}-m_{j}^{2}}=E-m_{j}^{2} / 2 E+\ldots$ and hence obtain for the neutrino mass eigenstate at distance $L$ from the source

$$
\left|v_{j}(L)\right\rangle=\mathrm{e}^{-i \frac{m_{j}^{2}}{2 E} L}\left|v_{j}(0)\right\rangle \text {. }
$$

One concludes, that the quantity

$$
H_{e f f}=\frac{M^{2}}{2 E}
$$

plays the role of effective Hamiltonian for the neutrino mass state.

While neutrino propagates in the mass state, it is produced and absorbed in the flavor state: only this state is observable. The neutrino emerges in a weak processes as a particular flavor state, then recasts into the mass eigenstates which propagate for some distance and must be recast back into the flavor eigenstates to describe the neutrino detection by weak interactions. This way neutrino changes its flavor. The transition amplitude of neutrino $v_{\alpha}$ to neutrino $v_{\beta}$ is given by

$$
A(\alpha \rightarrow \beta)=\sum_{j}\left\langle v_{\beta} \mid v_{j}(L)\right\rangle\left\langle v_{j}(0) \mid v_{\alpha}\right\rangle=\sum_{j}\left\langle v_{\beta} \mid v_{j}\right\rangle \mathrm{e}^{-i \frac{m_{j}^{2}}{2 E} L}\left\langle v_{j} \mid v_{\alpha}\right\rangle=\sum_{j} U_{\beta j} \mathrm{e}^{-i \frac{m_{j}^{2}}{2 E} L} U_{\alpha j}^{*} .
$$

Consequently, the transition probability reads

$$
\begin{aligned}
P\left(v_{\alpha} \rightarrow v_{\beta}\right) & =|A(\alpha \rightarrow \beta)|^{2} \\
& =\delta_{\alpha \beta}-4 \sum_{j>i} \operatorname{Re}\left[U_{\alpha j}^{*} U_{\beta j} U_{\alpha i} U_{\beta i}^{*}\right] \sin ^{2}\left(\frac{\Delta m_{j i}^{2}}{4 E} L\right)+2 \sum_{j>i} \operatorname{Im}\left[U_{\alpha j}^{*} U_{\beta j} U_{\alpha i} U_{\beta i}^{*}\right] \sin \left(\frac{\Delta m_{j i}^{2}}{2 E} L\right),
\end{aligned}
$$

where we introduced the squared mass differences $\Delta m_{j i}^{2} \equiv m_{j}^{2}-m_{i}^{2}$. They, but not the masses themselves, determine the transition.

In many cases the transition involves not all the three but only two states. Then equation (2) gets simplified. In $2 \leftrightarrow 2$ approximation to neutrino oscillations the transition probability (probability to change the neutrino flavor) becomes

$$
P\left(v_{\alpha} \rightarrow v_{\beta \neq \alpha}\right)=\sin ^{2} 2 \theta \cdot \sin ^{2}\left(\frac{\Delta m^{2}}{4 E} L\right),
$$

while the survival probability reduces to

$$
P\left(v_{\alpha} \rightarrow v_{\alpha}\right)=1-\sin ^{2} 2 \theta \cdot \sin ^{2}\left(\frac{\Delta m^{2}}{4 E} L\right)
$$


The typical distance characterizing the flavor change is oscillation length,

$$
L_{o s c}=\frac{4 \pi E}{\Delta m^{2}}=(2.5 \mathrm{~km}) \cdot \frac{E}{\mathrm{GeV}} \frac{\mathrm{eV}^{2}}{\Delta m^{2}} .
$$

There is also so-called matter effect, which may distort the introduced above vacuum oscillations, when neutrino propagates in a medium, filled with particles participating in the weak interactions. The classical example is electron neutrino produced by nuclei fusion in the Sun. These solar neutrinos propagate in the medium highly populated by electrons. The Fermi interaction

$$
\mathcal{L}=-2 \sqrt{2} G_{F} \bar{v}_{e} \gamma^{\mu} e \cdot \bar{e} \gamma_{\mu} v_{e}
$$

without macroscopic currents, $\left\langle\left\langle\bar{e}_{k} \gamma_{k l}^{i} e_{l}\right\rangle\right\rangle=0$, but with high density of electrons, $\left\langle\left\langle\bar{e}_{k} \gamma_{k l}^{0} e_{l}\right\rangle\right\rangle=$ $\left\langle\left\langle e^{\dagger} e\right\rangle\right\rangle=n_{e}$, yields after averaging $\left\langle\left\langle e_{k} \bar{e}_{l}\right\rangle\right\rangle=-\frac{1}{4} \gamma_{k l}^{0} \cdot n_{e}$ the neutrino-medium interaction term,

$$
\mathcal{L}_{e f f}=-\sqrt{2} G_{F} n_{e} \bar{v}_{e} \gamma^{0} v_{e},
$$

It gives the effective Hamiltonian in neutrino sector,

$$
V=\sqrt{2} G_{F} n_{e}
$$

to compete with the vacuum Hamiltonian(1). At sufficiently small energies the latter dominates, which gives rise to the vacuum oscillations, while the weak interactions are feeble. At larger energies the Hamiltonian (1) decreases, so that (3) governs the neutrino propagation, because the weak interaction grows with energy. In fact, electron neutrinos of high energies do not oscillate inside the Sun: the weak interactions are sufficiently fast to prevent this processes by collapsing the neutrino wave function back into the flavor basis. The process is known as Mikheev-Smirnov-Wolfenstein effect. The corresponding change in electron neutrino survival probability with energy has been observed by experiment BOREXINO [1].

To explain the neutrino oscillations, one must make neutrino massive, which implies departure from the SM. So far neutrino oscillations give the only direct evidence for the need of the physics beyond the SM.

\section{Sterile neutrinos}

One of the possible ways to explain the neutrino oscillations within the renormalizable extension of the SM is introducing sterile neutrinos. These are fermions singlet with respect to the SM gauge group (and hence sterile), which mix with the SM (or active) neutrinos. The mixing results in active neutrino masses and mixing responsible for the neutrino oscillation phenomena. Sterile neutrinos form right-handed neutrino components making the SM matter structure fully symmetric with respect to left-right transformations.

With sterile neutrinos one can explain the smallness of the active neutrino masses: why the neutrino scale is much lower than scales of all other electrically charged fermions of the SM, which masses range from $0.5 \mathrm{MeV}$ for electron to $173 \mathrm{GeV}$ for top-quark. The corresponding scheme is called seesaw type I mechanism [2-6] and works as follows.

Consider one sterile neutrino $N$ and one neutrino flavor, let it be electron. To the SM lagrangian one adds the new terms

$$
\mathcal{L}_{N}=\bar{N} i \not \supset N-f \bar{L}_{e}^{c} \tilde{H} N-\frac{M_{N}}{2} \bar{N}^{c} N+\text { h.c. },
$$


and we assume that the sterile neutrino mass $M_{N} \gg 1 \mathrm{eV}$, which is much bigger than the active neutrino masses, constrained from above by particle physics experiments and cosmological observations. Sterile neutrino mass is of Majorana type, so we introduce only two degrees of freedom. When the SM Higgs doublet $H$ acquires non-zero vacuum expectation value $v / \sqrt{2}$, the Yukawa term in (4), which includes SM lepton doublet $L_{e}$ with left electron and neutrino and $\tilde{H}_{i} \equiv \epsilon_{i j} H_{j}^{*}$ with $\epsilon_{i j}, i, j=1,2$ being fully antisymmetric $2 \times 2$ matrix, yields mass mixing in the neutral fermion sector,

$$
\mathcal{V}_{N}=\frac{1}{2}\left(\bar{v}_{e}, \bar{N}^{c}\right)\left(\begin{array}{cc}
0 & v \frac{f}{\sqrt{2}} \\
v \frac{f}{\sqrt{2}} & M_{N}
\end{array}\right)\left(\begin{array}{l}
v_{e} \\
N
\end{array}\right)+\text { h.c. }
$$

For the chosen hierarchy $M_{N} \gg M^{D}=v \frac{f}{\sqrt{2}}$ we have the flavor state

$$
v_{e}=U v_{1}+\theta N
$$

with $U \approx 1$ and active-sterile mixing

$$
\theta=\frac{M^{D}}{M_{N}}=\frac{v f}{2 M_{N}} \ll 1
$$

The mass eigenvalues are

$$
\approx M_{N} \text { and }-m_{a}=\theta^{2} M_{N} \lll M_{N}
$$

which naturally explains the smallness of neutrino masses by doubling the small parameter $\theta$. Note, that neither its value, nor the value of the sterile neutrino mass are fixed by the seesaw requirement to explain the active neutrino masses. In terms of the parameters of largangian (4) it means, that one and the same value of the active neutrino mass we obtain with larger Yukawas and heavier sterile neutrinos or with smaller Yukawas and lighter sterile neutrinos. In other words, the mass scale of sterile neutrinos is not fixed by the success of explaining the active neutrino masses within the framework of the seesaw mechanism.

The scheme above is straightforwardly extended to the case of several sterile neutrinos. To explain the presently confirmed results of neutrino oscillation experiments at least two non-zero active neutrino masses are needed, which asks for at least two sterile neutrinos. It looks weird to have the third active neutrino massless. If all the three are massive, one must introduce three or more sterile neutrinos, which allows us to address the issue of dark matter (see Ref. [7] for recent review on sterile neutrino dark matter).

\section{Sterile neutrino as dark matter}

Take sterile neutrino involved into the seesaw mechanism. Then its Majorana mass $M_{N}$ and Yukawa coupling $f$ in (4) are related to active neutrino mass $m_{a}$ as

$$
m_{a} \sim \frac{f^{2} v^{2}}{M_{N}^{2}} M_{N} \sim \theta^{2} M_{N} .
$$

Sterile neutrino mixes with active neutrinos, and hence, being (much) heavier, is always unstable: its decay into three active neutrinos, $N \rightarrow 3 v$, is kinematically allowed. To form dark matter, sterile neutrino lifetime must exceeds the age of the present Universe, $14 \mathrm{Gyr}$, that places constraint on the model parameters ${ }^{1}$,

$$
\theta^{2}<3 \times 10^{-3}\left(\frac{10 \mathrm{keV}}{M_{N}}\right)^{5}
$$

\footnotetext{
${ }^{1}$ Hereafter we consider the sterile neutrino lighter than the weak bosons, $M_{N} \ll 100 \mathrm{GeV}$, and adopt the Fermi effective theory for the weak processes.
} 
Plugging seesaw relation (6) into this inequality (7) we obtain for the sterile neutrino lifetime with respect to decay into three active neutrinos,

$$
\tau_{N \rightarrow 3 v} \sim 1 /\left(G_{F}^{2} M_{N}^{5} \theta^{2}\right) \sim 1 /\left(G_{F}^{2} M_{N}^{4} m_{v}\right) \sim 10^{11} \mathrm{yr}\left(10 \mathrm{keV} / M_{N}\right)^{4}
$$

The obtained results severely constrain the mass scale of sterile neutrino candidate for dark matter: only $\mathrm{keV}$ region turns to be suitable. Note that lighter sterile neutrinos do not fit to the dark matter phase space density, see e.g. [8].

Even if very long-lived, such dark matter is unstable, that provides a unique opportunity to search for its decay products. The golden mode is one-loop radiative decay $N \rightarrow \gamma v$, which goes through the loop of charged lepton and $W$-boson (sterile neutrino mass state emits them because of tiny, suppressed by $\theta \ll 1$, admixture of active neutrino flavor state (5)) with photon outgoing from either of the virtual particles. This is two-body decay into massless states, so in the neutrino rest frame the photon energy is exactly half of the neutrino mass, $E_{\gamma}=M_{N} / 2$. For sterile neutrino of Galactic dark matter the produced photons would form a narrow line, which relative width $\sim 10^{-3}$ is determined by the Doppler effect due to dark matter velocity dispersion in the Galaxy. The signature is very pronounced, and extensive investigations of Galactic $X$-ray spectrum and spectra of nearby dwarf and normal galaxies, galaxy clusters, put very strong limit on the sterile neutrino mixing angle (see e.g. [7]),

$$
\frac{\theta^{2}}{10^{-11}} \lesssim\left(\frac{10 \mathrm{keV}}{M_{N}}\right)^{4}
$$

surpassing that from the Universe age (7) by almost eight orders of magnitude. This limit is obtained assuming the sterile neutrino form all the dark matter component we needed in cosmology. If only a fraction of dark matter is due to sterile neutrinos, the limit becomes weaker proportionally to the fraction.

About three years ago two groups analyzing the data of Chandra telescope announced a feature consistent with the expected signal from decays of sterile neutrino dark matter $[9,10]$. The feature was found in stacking signal from many galaxies in Perseus cluster and in nearby Andromeda galaxy. Though the signal was recognized only at about $3 \sigma$ level, and at that level was missed in stacking signal from some other sets of galaxies, it nevertheless excited community which intensified hunting the sterile neutrino dark matter. The reason was that the model parameters, sterile neutrino mass and sterile-active mixing angle, were consistent with predictions of one of the realistic mechanisms of sterile neutrino production in the early Universe due to oscillations in primordial plasma.

To calculate the sterile neutrino production one can start with the Boltzmann equation for the sterile neutrino distribution function $f_{S}(t, \mathbf{p})$. In the Universe expanding at the Hubble rate $\mathcal{H}$ the Boltzmann equation reads (see e.g. [11])

$$
\frac{\partial}{\partial t} f_{s}(t, \mathbf{p})-\mathcal{H} \mathbf{p} \frac{\partial}{\partial \mathbf{p}} f_{s}(t, \mathbf{p})=\Gamma_{\alpha} P\left(v_{\alpha} \rightarrow v_{s}\right) f_{\alpha}(t, \mathbf{p}),
$$

where $\Gamma_{\alpha} \sim G_{F}^{2} T^{4} E$ is the weak interaction rate in plasma. This interaction collapses neutrino wave function and thus fixes the produced sterile neutrino state. The transition probability in the plasma 
can be written as

$$
\begin{aligned}
& P\left(v_{\alpha} \rightarrow v_{s}\right)=\sin ^{2} 2 \theta_{\alpha}^{\mathrm{mat}} \cdot \sin ^{2}\left(\frac{t}{2 t_{\alpha}^{\mathrm{mat}}}\right), \\
& t_{\alpha}^{\mathrm{mat}}=\frac{t_{\alpha}^{v a c}}{\sqrt{\sin ^{2} 2 \theta_{\alpha}+\left(\cos 2 \theta_{\alpha}-V_{\alpha \alpha} \cdot t_{\alpha}^{v a c}\right)^{2}}}, \\
& \sin 2 \theta_{\alpha}^{\mathrm{mat}}=\frac{t_{\alpha}^{\mathrm{mat}}}{t_{\alpha}^{v a c}} \cdot \sin 2 \theta_{\alpha}, \quad t_{\alpha}^{v a c}=\frac{2 E}{M_{N}^{2}}
\end{aligned}
$$

with effective plasma potential for active neutrinos consisting of two terms (below we omit flavor and temperature dependent numerical coefficients in front of the terms),

$$
V_{\alpha \alpha} \sim-\# G_{F}^{2} T^{4} E+\# G_{F} T^{2} \mu_{L_{\alpha}},
$$

lepton-symmetric and lepton-asymmetric, respectively. The latter is essentially of the same nature as the term (3) governing the propagation of energetic electron neutrinos in the Sun, where only electrons, but not positrons, are present in the plasma. In (8) $\mu_{L_{\alpha}}$ is the chemical potential with respect to leptonic charge $L_{\alpha}$.

The first term in (8) refers to the standard cosmology, when the plasma consists of all particles and antiparticles relativistic at a given temperature. It contributes to effective neutrino mass in plasma and suppresses the oscillations. The production rate is low, and sterile neutrino never came to equilibrium in the primordial plasma. The mechanism is called Dodelson-Widrow or non-relativistic production [12]. The amount of produced sterile neutrinosis proportional to mixing squared angle. For a given sterile neutrino mass the requirement to form the dominant component of the dark matter fixes the value of mixing angle. This is a line on $\left(\theta^{2}, M_{N}\right)$ plane, and at present it is entirely forbidden by negative results of searches for a single line in $X$-rays and studies of matter perturbation evolution at dwarf galaxy scales, see Fig. 1.

The second term in (8) emerges in the primordial plasma with lepton asymmetry. If the letter is generated before the epoch of electroweak transition, the asymmetry is redistributed between baryons and leptons, so that both asymmetries are of the same order. In this case the second term in (8) is too small to play any role in sterile neutrino production. In specific extensions of the SM (e.g. $v$ MSM $[15,16])$ the asymmetry can be produced later, and then it can be much larger than the asymmetry in the baryon sector, reaching the values as large as $10^{-3}$, being constrained from the primordial Big Bang Nucleosynthesis and anisotropy of the Cosmic Microwave Background radiation. The second term in (8) is linear in the chemical potential and hence, depending on the sign of asymmetry, can cancel the impact of the first term exhibiting the resonance in oscillations by significant increase of the effective mixing angle in plasma. Thus, the same amount of sterile neutrinos can be generated with smaller vacuum mixing angle $\theta$ [17]. In this setup the production depends both on vacuum mixing angle and the amount of lepton asymmetry in the primordial plasma. Then the region in the model parameter space, where sterile neutrino can form the entire dark matter, is not a line, but rather a wide band placed below the line of non-resonant production, see Fig. 1. The extensive studies of $X$-ray satellites data significantly shrank the viable region of parameter space asking for new mechanism of sterile neutrino production in the early Universe.

\section{Sterile neutrino production in models with additional scalars}

The prediction of sterile neutrino production can be drastically changed by introducing a scalar field $\phi$ coupled to the sterile neutrino,

$$
\mathcal{L} \propto \phi \bar{N}^{c} N+\text { h.c. }
$$



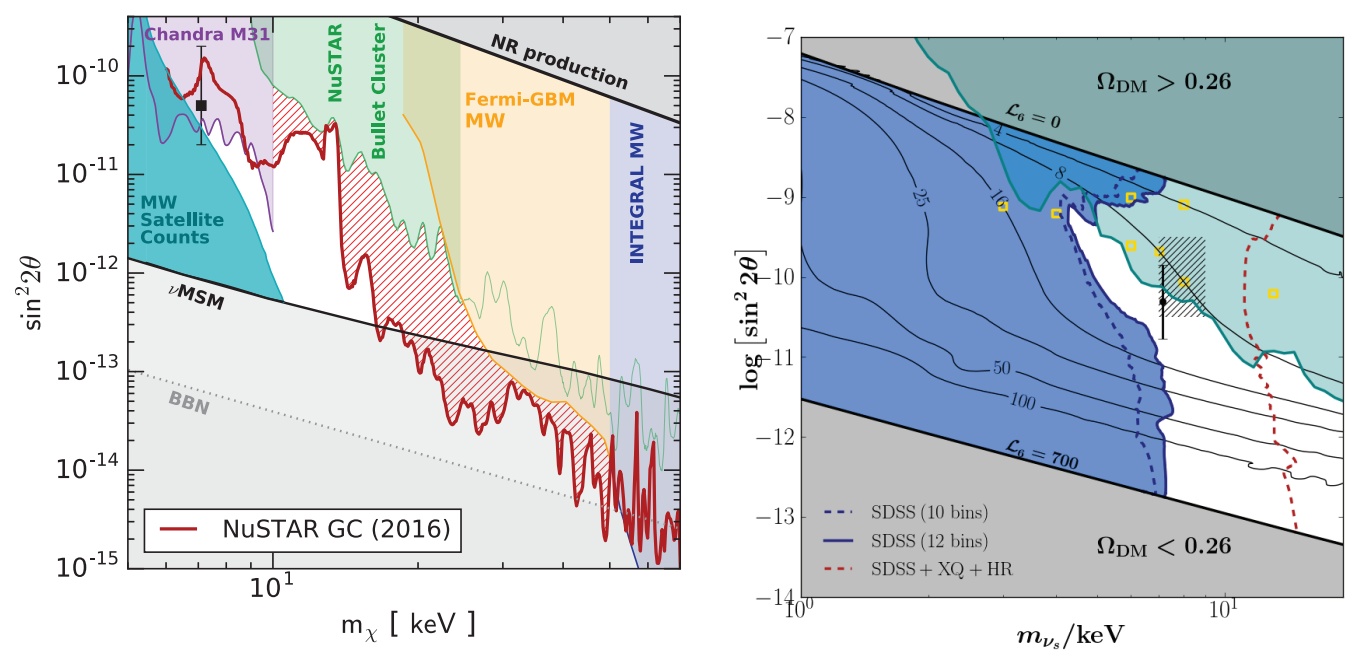

Figure 1. Present limits $[13,14]$ on the model parameters of sterile neutrino dark matter and predictions of dark matter production by non-resonant (lines 'NR production' and ' $\mathcal{L}_{6}=0$ ') and resonant oscillations in primordial plasma (with lepton asymmetry $\mathcal{L}_{6} \equiv L_{e} / 10^{6}$ ).

This coupling is well-motivated since it violates lepton symmetry being of the same nature as the Majorana mass term in largangian (4). Depending on the behavior of the scalar field in the early Universe, there are several possible impacts on the time-evolution of the sterile neutrino sector.

One of the options considered in Refs. $[18,19]$ is $\phi$ being the inflaton. Sterile neutrinos can indeed be produced by inflaton decays and can form dark matter. However, sterile-active mixing plays no role in this production mechanism, and all searches for sterile neutrinos aimed at measuring the mixing angle are irrelevant in this case.

Recently, two other options have been put forward [20], which allow for significant suppression of sterile neutrino production in the early Universe which still goes mostly through the active-sterile mixing. The first option exploits the very light free scalar field, which is frozen at high value in the early Universe. Then coupling (9) makes sterile neutrino superheavy in the early Universe, which kinematically suppresses all possible oscillations. The scalar remains frozen until the Hubble parameter drops to the value of the scalar mass. Then the field starts to oscillate with decreasing amplitude and by the present epoch the scalar contribution to the sterile neutrino mass (9) can be neglected. Some amount of sterile neutrinos can be produced in neutrino oscillations while the scalar amplitude drops. However, by choosing the scalar parameters one can make this amount as small as possible.

Another option is related to the scalar field of the hidden sector, which dynamics initiates the phase transition in the late Universe, so that $\phi$ gains non-zero vacuum expectation value. Then, with zero sterile neutrino mass term in lagrangian (4) one has massless sterile neutrino in the early Universe, and hence no sterile-active oscillations at all. Later, after the phase transition, sterile neutrino becomes massive and hence participating in neutrino oscillations. Since the non-resonant production mechanism is mostly efficient at temperature [12]

$$
T_{\max } \approx 133 \mathrm{MeV}\left(\frac{1 \mathrm{keV}}{M_{N}}\right)^{1 / 3}
$$


the phase transition must occur later, at lower temperature, which guarantees the suppression of sterile neutrino production in oscillations as compared to the non-resonant mechanism. The results are illustrated in Fig. 2.
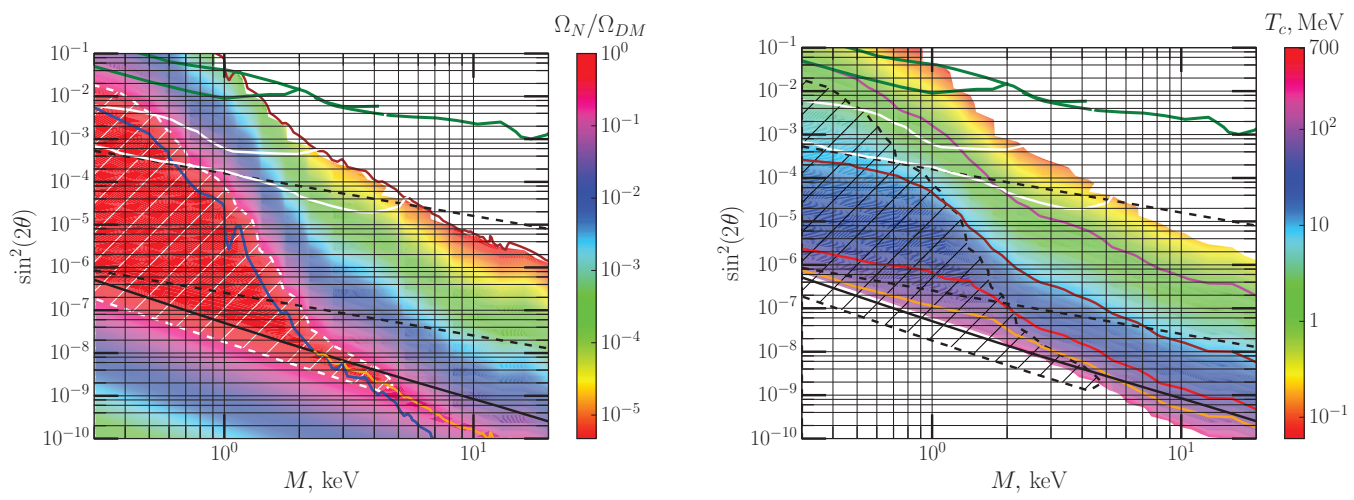

Figure 2. Dark matter sterile neutrino production in the extension with scalar from the hidden sector exhibiting the phase transition at temperature $T_{c}$, which makes sterile neutrino massive [8]. Green lines refer to present direct limits on sterile-neutrino mixing, while white lines indicate the prospect of future direct searches. The dotted black lines show the regions where the sterile neutrino can truly participate in seesaw mechanism providing active neutrinos with masses of viable scale. The limits from $X$-rays are avoided at relatively large and moderate mixings because of smaller sterile neutrino fraction in dark matter, $\Omega_{N} / \Omega_{D M}<1$. See Ref. [8] for more details.

While the suppression of sterile neutrinos in the early Universe makes models with moderate and even relatively large mixing angles consistent with cosmology, the price to be paid is the unsolved dark matter problem. The limits from X-rays are rather severe, and constrain the models parameters for any dark matter production in the early Universe. They can be avoided only assuming that the sterile neutrinos form only a (small) fraction of dark matter, $\Omega_{N}<\Omega_{D M}$. Then the $X$-ray bounds rescale as

$$
\theta_{X-\text { ray }}^{2}=\theta_{\alpha I}^{2} \frac{\Omega_{N}}{\Omega_{D M}}
$$

opening a possibility for the $\mathrm{keV}$-scale sterile neutrino to fully participate in seesaw mechanism and even being tested in future experiments on direct searches for light sterile neutrinos.

Note in passing that red region on left panel of Fig. 2, consistent with all bounds from astrophysics and direct searches, implies $\Omega_{N} \sim \Omega_{D M}$, so the sterile neutrinos form dominant dark matter component. However, sterile neutrinos are light here, and being produced in oscillations (hence with momenta $\sim 3 T$ ) happen to be too fast at the onset of structure formation in the early Universe. They would suppress the formation of protohalo for future dwarf galaxies, which be observe today, though at a bit smaller amount than one expects for the cold dark matter particles. The problem is solved if sterile neutrino contribution to dark matter is only sub-dominant here. To make this region cosmologically viable with sterile neutrino forming entire dark matter component, one can involve another production mechanism, where sterile neutrino would be cold. One of the possibility is to use the oscillating scalar field with coupling (9) as the neutrino source exploiting the first aforementioned option with initially frozen and then oscillating scalar $\phi$. 


\section{Conclusions}

Sterile neutrinos is one of the optional physics beyond the SM, which may be (partly) responsible for the dark matter phenomenon. Introduced to explain neutrino oscillations, light sterile neutrinos can be produced in the early Universe and are long-lived enough to survive until the present days and populate galaxy and galaxy clusters. While the minimal model was extensively studied and the viable parameter space shrank significantly, we show that natural modification of the model with additional scalar field (possibly playing a role of Majoron) opens up new room available even for direct searches and promising interesting cosmological and phenomenological consequences.

The work is supported by the RSF grant 17-22-01547.

\section{References}

[1] M. Agostini et al. (Borexino) (2017), 1709.00756

[2] P. Minkowski, Phys. Lett. B67, 421 (1977)

[3] M. Gell-Mann, P. Ramond, R. Slansky, Conf. Proc. C790927, 315 (1979), 1306.4669

[4] R.N. Mohapatra, G. Senjanovic, Phys. Rev. Lett. 44, 912 (1980)

[5] T. Yanagida, Prog. Theor. Phys. 64, 1103 (1980)

[6] J. Schechter, J.W.F. Valle, Phys. Rev. D22, 2227 (1980)

[7] M. Drewes et al., JCAP 1701, 025 (2017), 1602 . 04816

[8] D. Gorbunov, A. Khmelnitsky, V. Rubakov, JCAP 0810, 041 (2008), 0808 . 3910

[9] E. Bulbul, M. Markevitch, A. Foster, R.K. Smith, M. Loewenstein, S.W. Randall, Astrophys. J. 789, 13 (2014), 1402.2301

[10] A. Boyarsky, O. Ruchayskiy, D. Iakubovskyi, J. Franse, Phys. Rev. Lett. 113, 251301 (2014), 1402.4119

[11] D.S. Gorbunov, V.A. Rubakov, Introduction to the theory of the early universe: Hot big bang theory (World Scientific, Hackensack, 2011), http://www.DESY.eblib.com/patron/ FullRecord. aspx? $\mathrm{p}=737614$

[12] S. Dodelson, L.M. Widrow, Phys. Rev. Lett. 72, 17 (1994), hep-ph/9303287

[13] K. Perez, K.C.Y. Ng, J.F. Beacom, C. Hersh, S. Horiuchi, R. Krivonos, Phys. Rev. D95, 123002 (2017), 1609.00667

[14] J. Baur, N. Palanque-Delabrouille, C.Y. Eche, A. Boyarsky, O. Ruchayskiy, E. Armengaud, J. Lesgourgues (2017), 1706.03118

[15] T. Asaka, S. Blanchet, M. Shaposhnikov, Phys. Lett. B631, 151 (2005), hep-ph/0503065

[16] T. Asaka, M. Shaposhnikov, Phys. Lett. B620, 17 (2005), hep-ph/0505013

[17] X.D. Shi, G.M. Fuller, Phys. Rev. Lett. 82, 2832 (1999), astro-ph/9810076

[18] M. Shaposhnikov, I. Tkachev, Phys. Lett. B639, 414 (2006), hep-ph/0604236

[19] F. Bezrukov, D. Gorbunov, JHEP 05, 010 (2010), 0912 . 0390

[20] F. Bezrukov, A. Chudaykin, D. Gorbunov, JCAP 1706, 051 (2017), 1705. 02184 